\title{
Editorial
}

\section{Celebrating Peer Review Week 2017: Transparency in the Review Process}

This year JNeurosci will again participate in Peer Review Week, to be celebrated from the 11-17 of September (https://peerreviewweek. files.wordpress.com/2016/06/prw-press-release-2017.pdf). Last year we highlighted the incredible contributions of our volunteer reviewers (we can't do this without you!) by identifying a few outstanding individuals and letting them know how much we appreciate their contributions. This year our Reviewing Editors have once again identified some of the most responsive, rigorous, rapid reviewers, and we will highlight their contributions all this week. Also, I want to thank all our Reviewing and Senior Editors personally for the service that they provide to JNeurosci and our authors every day. It is not easy to risk the disappointment of peers to handle manuscripts rigorously and fairly. Thank you all for the work you do for the neuroscience community.

Our Associate Editors are brilliant reviewers and scientists, and we would like to thank them as well. We choose Associate Editors for our Editorial Board from among our most frequent and helpful reviewers, those who can point out the methodological and scientific strengths and weaknesses of a manuscript and put the findings into a larger context. We often call on them to advise us when two reviewers have opposing opinions on a paper, or when a reviewer who has accepted an assignment is very late with a review, and we need an opinion very quickly. We could not keep the peer review process running smoothly without our Associate Editors.

This year, the focus of Peer Review Week is on transparency of review. In keeping with this theme, we have prepared a video (https://bcove.video/2iZg0oC) pulling back the curtain on the review process at JNeurosci. Those of you who have served as editors of scientific journals probably have a very good idea of what goes on behind the scenes once a manuscript is submitted. However, many in our community have not yet served on an editorial board, and some trainees may not yet have served as a peer reviewer, so submitting a manuscript may seem like pushing it into a black box. The video follows the editorial process, step by step, from submission to decision. A link to this video will be on the JNeurosci website and will stay on the site as a resource for our authors after Peer Review Week. We are also hosting an open discussion on NeurOnLine this week to answer your questions about the peer review process. Please go to https://community.sfn.org/t/peer-reviewweek-open-forum/7736 for more information on this discussion with the editors of JNeurosci and eNeuro.

Please look for Tweets about our outstanding reviewers this week by following@MarinaP63 and @SfNJournals on Twitter. Although we can only highlight a small subset of our volunteer reviewers at this time of year, a list of all our reviewers and a special mention for our most frequent reviewers can be found on the JNeurosci website (http://www.jneurosci.org/content/ general-information\#reviewer). Thank you again for the work you do for JNeurosci and the neuroscience community.

As always, we are eager to hear from you on what we can do better. Please email me at JN_EIC@SFN.org or Tweet to @MarinaP63 with questions and comments.

(D) Marina Picciotto, EiC, JNeurosci

DOI:10.1523/JNEUROSCI.2345-17.2017 